\title{
Análise da eficiência do mercado futuro brasileiro de boi gordo usando co-integração
}

\author{
André Steffens Moraes ${ }^{1}$ \\ Ricardo Chaves Lima² \\ André de Souza Melo ${ }^{3}$
}

Resumo: A hipótese de que os preços futuros são preditores não viesados dos preços à vista é uma hipótese conjunta de que os mercados são eficientes e que não existe prêmio ao risco. Entretanto, na presença de prêmio ao risco, a hipótese de não viés pode ser rejeitada mesmo quando o mercado é eficiente. Este artigo testa a eficiência do mercado futuro brasileiro do boi gordo na presença de prêmio ao risco, usando técnicas de co-integração. Os resultados mostram que o mercado futuro do boi gordoé eficiente e não viesado no longo prazo, independente da presença de prêmio ao risco.

Palavras-chaves: mercado futuro do boi gordo, hipótese da eficiência de mercado, co-integração.

Abstract: The hypothesis that future prices are unbiased predictors of spot prices is a joint hypothesis that markets are efficient and risk premium are absent. However, the unbiasedness hypothesis may be rejected in the presence of a risk premium, even when the market is efficient. The objective of this article is to test market efficiency for the Brazilian live cattle while permitting the presence of risk premium, using cointegration techniques. Results show that the future markets for live cattle are efficient and unbiasedness in the long run, and does not depend of the presence of a risk premium.

Key-words: live cattle futures market, efficient market hypothesis, cointegration.

Classificação JEL: Q13, G14.

${ }^{1}$ Doutor em Economia pela Universidade Federal de Pernambuco (2008). Pesquisador da Embrapa Pantanal.E-mail: andre@cpap.embrapa.br

${ }^{2}$ Doutor em Economia Agrícola pela Universidade do Tennessee (1994). Professor Ajunto II do Depto de Economia da Universidade Federal de Pernambuco e professor convidado do Mestrado em Gestão Global da Universidade de Ciências Aplicadas de Bremen (Hochschule Bremen), na Alemanha. E-mail: chaveslima@gmail.com

${ }^{3}$ Mestre em Economia pela Universidade Federal de Pernambuco (2009). Doutorando em Economia na Universidade Federal de Pernambuco. E-mail: andredesouzam@gmail.com 


\section{Introdução}

Muitos agentes econômicos tomam decisões baseadas em hipóteses sobre eventos futuros, cuja incerteza pode ser reduzida por operações em mercados futuros, que oferecem um mecanismo de proteção contra os riscos dessas operações. Mas este mecanismo só é efetivo se o mercado é eficiente. Nesse caso, o preço futuro é um ótimo previsor do preço à vista na data de terminação do contrato. A única diferença admissível entre os dois sendo um erro aleatório não previsível de média zero (NEWBOLD et al., 1999b). A hipótese da eficiência de mercado (HEM) é uma hipótese conjunta de que os agentes econômicos formam suas expectativas de maneira racional e são neutros ao risco. Isto é, que os mercados são eficientes e não há prêmio ao risco. Isto implica que, em um mercado futuro eficiente, os preços futuros correntes, $F_{t}$, de um contrato para entrega de um produto no tempo $t+1$, podem ser considerados estimadores não viesados dos preços à vista futuros, $S_{t+1}$, pois incorporam toda a informação disponível para predizer este preço futuro (NEWBOLD et al., 1999b; BECK, 1994; FAMA, 1970).

Para testar a HEM têm sido estimadas várias versões de:

$$
S_{t+1}=c_{0}+c_{1} F_{t}+\mu_{t+1}
$$

em que $S_{t+1}$ é o preço no mercado físico (spot) no tempo $t+1$, $F_{t}$ é o preço corrente do contrato futuro, $c_{0}$ e $c_{1}$ são parâmetros e $\mu_{t}$ é um termo de erro aleatório (decorrente de se assumir expectativas racionais), que é independente e identicamente distribuído, com média zero e variância constante (i.é, é um processo de ruído branco). A hipótese nula é que o intercepto não é significantemente diferente de zero e que o coeficiente de inclinação não é significantemente diferente de um, ou seja, $c_{0}=0$ e $c_{1}=1$ (BECK, 1994).

Esta equação evidencia de uma forma simples os conceitos subjacentes à hipótese de eficiência de mercado, já que é possível rearranjá-la para:

$$
\begin{aligned}
& S_{t+1}-c_{1} F_{t}=c_{0}+\mu_{t}+1 \\
& \text { Se } c_{0}=0 \text { e } c_{1}=1, \text { então } \\
& S_{t+1}-F_{t}=\mu_{t+1}
\end{aligned}
$$

Tomando a expectância da Equação (3), tem-se:

$$
E_{t}\left(S_{t+1}-F_{t}\right)=0
$$

Os preços descritos acima são usualmente referidos como um passeio aleatório (random walk), pois seus movimentos (ou mudanças) futuras não podem ser previstos porque, dado o valor de hoje, eles podem tanto subir como descer. 
Como resultado, os preços podem se desviar substancialmente de seus valores iniciais, e pode ser necessária a ocorrência de um grande número de observações antes que eles retornem aos seus valores iniciais. Além disso, a mudança média esperada nos preços é zero e, como os erros são não-correlacionados, quaisquer mudanças nos preços também serão não-correlacionadas.

Entretanto, devido à característica típica de não-estacionariedade das séries temporais de preços, quando se estima uma regressão do tipo da Equação (1), é possível que se obtenham resultados espúrios (GRANGER e NEWBOLD, 1974). Uma forma de prevenir este problema é verificar se as variáveis incluídas no modelo econométrico são co-integradas. A existência de co-integração entre duas séries de preço assegura que elas exibam um comportamento compatível no longo prazo. A co-integração implica que os resíduos das relações de longo prazo são estacionários, uma condição necessária mas não suficiente para a eficiência. Para os mercados futuros serem considerados eficientes é também requerido que os erros sejam ruído branco; se forem autocorrelacionados, a informação dos preços passados pode ser usada para predizer os preços à vista futuros e isto contradiz o requerimento de que os preços futuros instantânea e completamente refletem toda a informação disponível (NEWBOLD et al., 1999b).

O mercado futuro do boi gordo da Bolsa de Mercadorias e Futuros (BM\&FBovespa) tem mostrado um grande dinamismo nos últimos anos, com uma evolução que se mantém a frente de outros produtos agropecuários. Este mercado teve destaque nos oito primeiros meses de 2006 entre os mercados agropecuários da BM\&FBovespa: fechou agosto com 48.755 contratos, $25 \%$ a mais que o mesmo mês de 2005 e 18,8\% acima de julho/06. O recorde histórico de posições em aberto nesse mercado foi alcançado no dia 30 de agosto de 2006, com 32.281 contratos, sendo a média diária negociada neste mês de 42.413 cabeças/dia. No acumulado de janeiro a agosto de 2006, os contratos de boi gordo também alcançaram recorde histórico, com 201.586 contratos negociados, movimentando cerca de US\$ 1,7 bilhão (BM\&FBOVESPA, 2006). Os negócios com boi gordo na BM\&FBovespa cresceram 98,5\% em 2004, o maior desempenho entre os derivativos agropecuários negociados no ano, atingindo o total de 225.200 contratos. Em 2005, o número de contratos negociados mensalmente teve o crescimento mais expressivo em relação aos três anos anteriores. Apesar disso, no total de negócios da Bolsa vem representando apenas cerca de $10 \%$ do total anual de abate de bovinos no Brasil. Essa pequena participação indica o potencial de crescimento deste mercado no futuro (MARTIN e BEZERRA, 2005).

O objetivo deste artigo é testar a eficiência do mercado futuro brasileiro do boi gordo na presença de um prêmio ao risco. A seção 2 faz uma revisão bibliográfica sobre a eficiência de mercado de produtos agrícolas, com destaque para a literatura que se detém no mercado do boi gordo. A seção 3 descreve os dados e os testes de eficiência baseados na teoria da co-integração. A seção 4 apresenta os resultados empíricos e os discute. A seção 5 conclui. 


\section{Revisão Bibliográfica}

Fama (1970) desenvolveu originalmente a teoria da eficiência de mercado, e sua definição ficou conhecida como a hipótese de eficiência de mercado (HEM). Segundo Fama (1970), um mercado eficiente é aquele que incorpora acuradamente toda a informação conhecida na determinação do preço, de modo que o preço sempre reflete toda informação disponível em um mercado eficiente. As únicas mudanças de preço que podem ocorrer são aquelas que resultam de nova informação. Em um mercado eficiente, ninguém deve ser capaz de obter lucro econômico anormal usando a informação disponível, significando que não existem oportunidades de lucro a serem exploradas aos preços vigentes. Se os agentes são racionais, então as suas expectativas sobre os preços futuros são iguais às previsões ótimas usando todas as informações disponíveis. Embora existam consideráveis desacordos sobre o grau no qual a HEM se mantém, ela se tornou o paradigma dominante usado pelos economistas para compreender e investigar o comportamento dos mercados financeiros e de produtos.

A eficiência dos mercados futuros tem sido um dos tópicos mais pesquisados e mais controversos na literatura empírica sobre eficiência de mercado: alguns autores encontram evidência de eficiência, outros de ineficiência, sugerindo que a hipótese de eficiência é suportada somente para certos mercados e somente para alguns períodos. Ver, por exemplo, Fama (1970), Leuthold (1974), Kellard et al. (1999). Já os artigos de Beck (1994), Chu et al. (1999) e Ackert e Racine (1999) encontraram evidência consistente com a eficiência dos mercados futuros. Embora a evidência da literatura pareça ser decididamente mista, é possível que algumas conclusões sejam reflexo da falta de atenção dada aos aspectos institucionais que governam o funcionamento dos mercados futuros e às características estatísticas específicas das séries temporais de preços de produtos, as mais relevantes das quais, segundo Newbold et al. (1999a), são a sazonalidade, a sobreposição de dados e as observações desigualmente espaçadas. Para Newbold et al. (1999a) a grande quantidade de resultados divergentes produzidos é altamente dependente das técnicas econométricas adotadas e do período de tempo analisado. Crowder e Phengpis (2003), além do período da amostra e da técnica utilizada, acrescentam que a forma fraca também é dependente do produto agrícola testado e do tipo de dados. As rejeições da eficiência têm sido interpretadas argumentando-se que os agentes econômicos não processam racionalmente a informação disponível e que o preço futuro é um estimador viesado devido à presença de um prêmio ao risco constante ou variando no tempo (NEWBOLD et al., 1999a, 1999b; GARCIA et. al, 2004). Malkiel (2003) sumariza a questão argumentando que, de uma maneira geral, os resultados dos testes de eficiência de aleatoriedade e habilidade de previsão são consistentes com a visão dos economistas de que, no longo prazo, os mercados 
futuros são eficientes, mas que no curto prazo podem existir ineficiências em certos períodos.

Newbold et al. (1999a) testaram a eficiência dos mercados futuros de cinco produtos agrícolas no contexto de contratos desigualmente espaçados. Tal característica, resultante da natureza sazonal da produção e dos aspectos institucionais do mercado, requer o desenvolvimento de técnicas econométricas apropriadas, já que as técnicas padrões de séries temporais só podem ser aplicadas quando as observações são igualmente espaçadas. Os autores argumentam que desconsiderar esta característica pode conduzir a conclusões incorretas e inexatas sobre a eficiência de mercado. Assim, a diversidade de resultados existente na literatura pode refletir uma falta de atenção às propriedades específicas das séries de dados e às características institucionais do mercado (NEWBOLD et al., 1999b).

Em sua definição original da HEM, Fama (1970) assumiu ausência de custos de transação (como taxas de corretagem) e de obtenção da informação, o que é irrealista. A existência desses custos implica uma definição alternativa da eficiência de mercado: um mercado é eficiente com relação à informação disponibilizada no tempo $\mathrm{t}$ se os retornos econômicos gerados pelas operações usando esta informação não excedem os custos de transação e de aquisição da informação (ZULAUF e IRWIN, 1997). Com relação ao modelo de eficiência de mercado de Fama (1970), implica que $c_{1}$ pode ser diferente de um porque o mercado adquire e analisa a informação lentamente, mas os operadores possuem maior habilidade para adquirir primeiro a informação. Assim, os preços já não refletem perfeitamente toda a informação disponível. Essas violações da hipótese de Fama (1970), chamadas anomalias, trouxeram dúvidas quanto à validade dessa hipótese (ZULAUF e IRWIN, 1997).

Se a condição de eficiência de preços (o preço futuro é um estimador não viesado do preço à vista) não é satisfeita, uma fonte de erro é a presença de um prêmio ao risco. Muitos estudos nos anos 1960 se concentraram no conceito de prêmio ao risco, sobressaindo a conclusão que a existência de um erro nos preços relacionado ao prêmio ao risco era muito improvável (GRAY e RUTLEDGE, 1971). Em estudos posteriores, os resultados parecem ter sido influenciados pelos procedimentos usados e pelo prêmio ao risco assumido. Por exemplo, Fama e French (1987), estudando vários produtos agrícolas, concluem que há pouca evidência da existência de um prêmio ao risco constante. Entretanto, McKenzie e Holt (2002), usando co-integração e modelo de correção de erros com processo $\mathrm{ARCH}$, encontraram prêmios ao risco variando no tempo para porco e boi gordo, mas não para os outros produtos analisados (milho e soja).

Para Beck (1994), assumir que os participantes do mercado são neutros ao risco (implícito na hipótese de ausência de prêmio ao risco), não é defensável teoricamente nem plausível empiricamente. Este autor argumenta que os 
produtores aversos ao risco demandam contratos futuros para proteger seus produtos e, assim, criam um prêmio ao risco que irá viesar os preços futuros, de modo que $c_{0} \neq 0$. Além disso, Danthine (1978) mostrou que, quando os produtores são aversos ao risco, a otimização intertemporal resulta tanto em $c_{0} \neq 0$ quanto em $c_{1} \neq 1$, mesmo em mercados eficientes. Logo, dado que existe um prêmio ao risco, os testes de eficiência não dependem da ausência desse prêmio.

Zulauf e Irwin (1977) afirmam que um mercado futuro pode ser eficiente em termos da definição de Fama (1970) e ter um viés de preço (ou seja, $E_{t}\left(S_{t+1}-F_{t}\right) \neq 0$, Equação (4)). Dado que o erro é uma compensação pelo risco, quando existe um risco que não pode ser diversificado, a atividade associada com esse risco deve receber um retorno superior ao retorno livre de risco. Esse viés no preço pode ser denotado como $c_{0} \neq 0$, sendo $c_{0}$ a compensação pelo risco. Haveria, assim, duas versões da eficiência de Fama (1970): uma no qual $c_{0}=0$ e $c_{1}=1$, e outra em que $c_{0}$ $\neq 0$ e $c_{1} \neq 1$, dado que um $c_{0}$ não zero é um retorno ao risco. Esses vieses de preço podem ou não ser constantes no tempo. Zulauf e Irwin (1997), concluem que, apesar da existência de viés de preço ser extremamente controversa, sua existência é uma questão empírica, e não uma questão conceitual.

Bessler e Covey (1991) aplicaram a formulação de co-integração para examinar as relações entre preços diários futuros e à vista no mercado de boi gordo dos EUA, com resultados mistos, isto é, evidência limitada de relações de longo prazo entre preços futuros e à vista para contratos futuros de curta terminação; para contratos de terminação mais distante, não foram encontradas relações de co-integração. Leuthold (1979) também encontrou ausência de co-integração entre os preços à vista e contratos futuros de terminação distante em mercados de boi gordo: cotações de preços futuros entre 4 e 8 meses antes da maturação são estimadores viesados dos preços à vista do boi gordo.

McKenzie e Holt (2002) testaram a eficiência dos mercados futuros dos EUA para cinco produtos agrícolas, entre os quais boi gordo. Seus resultados indicaram que os mercados futuros para todos os produtos, exceto um, eram eficientes e não viesados no longo prazo, mas o mercado de boi gordo exibiu ineficiência e viés no curto prazo e mostrou um prêmio ao risco variando no tempo. Kellard et al. (1999) analisaram a eficiência da previsão dos mercados de soja, porco e boi gordo e concluíram que esses mercados são eficientes no longo prazo, mas encontraram ineficiências no curto prazo, particularmente para o boi gordo.

Newbold et al. (1999b) investigaram os efeitos da sazonalidade nos testes de eficiência de vários produtos agrícolas, entre os quais boi gordo, particularmente a extensão na qual padrões sazonais fortes e antecipados podem ser responsáveis pela ineficiência encontrada em mercados futuros. Os autores encontraram evidência de que os termos sazonais são significantes, sugerindo a presença de ineficiências de mercado, já que a informação sobre o padrão sazonal não é 
incorporada na base e pode ser usada pelos agentes para predizer os movimentos dos preços à vista futuros. Os resultados mostraram que o mercado de boi gordo é muito ineficiente em curtos e longos horizontes de previsão, mas o efeito sazonal parece ser apenas marginalmente responsável por esta ineficiência.

No Brasil, o mercado do boi gordo foi instituído pela Bolsa Mercantil de São Paulo em 1981. Relançado em 1991 pela BM\&FBovespa, passou por várias reestruturações, principalmente em seu desenho contratual e em seus padrões internos de negociação. Lazzarini et al. (1998) relatam a história do mercado futuro do boi gordo na BM\&FBovespa e os problemas que surgiram na negociação de seus contratos, discutindo os fatores de sucesso ou fracasso com base em um estudo de caso. Esses autores testaram a influência de determinados fatores na demanda pelo contrato futuro de boi gordo na BM\&FBovespa, com ênfase em duas alterações contratuais: liquidação do contrato por entrega física em um único curral (em contraposição a até então vigente entrega física em vários currais) e liquidação financeira mediante um índice de preços (Indicador de preço ESALQ/BM\&FBovespa), eliminando a entrega física (desenho contratual que permanece até hoje). O modelo econométrico levou em consideração indicadores de sucesso e liquidez dos contratos e de variabilidade dos retornos no mercado físico. Os resultados mostraram que a liquidez defasada do mercado influencia o nível de contratos em aberto, com maior demanda por contratos futuros à medida que os custos de transação são reduzidos. Os resultados também evidenciaram que um aumento na variabilidade dos preços no mercado físico tende a aumentar a demanda por contratos futuros. Além disso, os efeitos positivos das duas alterações contratuais indicaram que o desenho dos contratos é o aspecto que mais influencia a demanda por contratos futuros, sendo a redução dos custos de transação ex post o principal fator responsável pelo aumento na demanda dos contratos.

Bressan (2004) analisa a aplicabilidade de modelos de previsão de séries temporais como ferramenta de decisão na compra e venda de contratos futuros de boi gordo, café e soja na BM\&FBovespa, em datas próximas ao vencimento. Utilizando modelos univariados de previsão de preços (ARIMA, redes neurais artificiais, e modelos lineares dinâmicos), e com base em cotações semanais dos mercados físico e futuro, o autor calculou os retornos médios desses produtos no mercado futuro, indicando o potencial de utilização desses modelos como ferramenta de decisão. Para comparação, foi utilizado o índice de Sharpe. A maioria dos contratos apresentou retornos financeiros positivos, com destaque para operações fundamentadas nas previsões dos modelos lineares dinâmicos e ARIMA, mas com diferenças no desempenho preditivo. 


\section{Metodologia}

O conceito de co-integração foi introduzido por Engle e Granger (1987) e tem sido amplamente empregado na análise de séries temporais. Este conceito corresponde à noção estatística da existência de um relacionamento a longo prazo entre as variáveis econômicas. Assim, duas ou mais variáveis são co-integradas quando existe uma combinação linear entre elas que seja estacionária, embora as variáveis não o sejam individualmente. Estatisticamente, uma série temporal é estacionária quando sua média, variância e covariância não variam no tempo. Neste caso, a série é denotada por I(0), significando que ela é integrada de ordem zero. A ordem de integração é o número de vezes que uma variável precisa ser diferenciada para atingir a estacionariedade. Uma série que precisa ser diferenciada uma vez para atingir a estacionaridade é denotada por I(1). Geralmente, qualquer combinação linear de duas séries não-estacionárias I(1) também será I(1). No entanto, se existir alguma combinação linear entre essas duas séries temporais que seja I(0), então, existirá co-integração entre as duas séries.

As técnicas de co-integração desenvolvidas por Engle e Granger (1987) podem testar a eficiência de mercado na presença de prêmio ao risco. Nesse caso, rejeitar a hipótese nula de não viés significa rejeitar a eficiência, dada a forma assumida do prêmio ao risco. O procedimento usual envolve dois passos. Primeiro, examinar a relação de co-integração (condição necessária) entre as duas séries de preço $S_{t}$ e $F_{t-1}$. Se existe co-integração, testam-se restrições nos parâmetros, $c_{0}=0$ e $c_{1}=1$, já que a eficiência de mercado também requer uma previsão não viesada dos preços futuros sobre os preços à vista. O segundo passo pode consistir de testes múltiplos: $c_{0}=0$ e $c_{1}=1$ conjuntamente ou cada um individualmente. A regressão de co-integração é usualmente especificada como a Equação (1). Se as séries são co-integradas e, ao menos, a segunda restrição não é rejeitada, então é possível testar a hipótese de que a diferença entre o preço à vista e o preço futuro no longo prazo é devida somente a uma média constante. A restrição $c_{1}=1$ é o indicador mais importante de eficiência de mercado, porque $c_{0}$ pode ser diferente de zero sob a existência de prêmio ao risco mesmo quando o mercado é eficiente. A relação de co-integração e as restrições nos parâmetros podem ser testadas usando a abordagem de Johansen (1988) e o teste de razão de verossimilhança, respectivamente. Quando a regressão envolve apenas duas variáveis, o teste de co-integração de Granger pode ser usado ao invés do teste de Johansen (NEWBOLD et al., 1999b).

É possível realizar um teste que permita a presença de um prêmio ao risco, isto é, que não imponha as hipóteses $c_{0}=0$ e $c_{1}=1$. Neste caso, são testadas mais restrições do que no teste anterior, sendo, portanto, um teste de eficiência mais forte. Mesmo neste caso é possível que os resíduos sejam serialmente 
correlacionados, o que viola a eficiência de mercado, pois implica que os preços à vista dependem não só dos preços futuros correntes, mas, também, dos preços passados (BECK, 1994). Para efetuar este teste é necessário transformar as séries co-integradas para uma representação de correção de erro (GRANGER, 1986):

$$
\Delta S_{t-1}=a-\rho \mu_{t}+b \Delta F_{t}+\sum_{i=1}^{m} \beta_{i} \Delta S_{t+1-i}+\sum_{i=1}^{m} \gamma_{i} \Delta F_{t-i}+\varepsilon_{t+1}
$$

ou

$$
S_{t-1}=(1-\rho) S_{t}+b F_{t}+\left(\rho c_{1}-b\right) F_{t-1}+\rho c_{0}+\varepsilon_{t+1}
$$

em que $\varepsilon_{t+1}$ é uma série estacionária possivelmente serialmente correlacionada com média zero e variância constante e na Equação (6) $\mu_{t+1}$ foi substituído por $S_{t+1}-c_{0}-c_{1} F_{t}$ (com os termos defasados omitidos, para maior clareza). As séries transformadas são agora estacionárias, de modo que os coeficientes estimados têm distribuição assintótica normal, permitindo testar a hipótese de que $c_{0}=0$ e $c_{1}=1$. A condição de que os erros não sejam correlacionados é testada com a estatística $Q$, de Ljung=Box, aplicada aos resíduos $\mu_{t+1}$.

A co-integração requer que $\rho>0$ para que desvios no equilíbrio de longo prazo (Equação (1)), se reflitam nos preços à vista. Para eficiência de mercado é necessário que $\rho=1, \rho c_{1}=\mathrm{b} \neq 0$ e $\beta_{i}=\gamma_{i}=0$. A eficiência de mercado também implica que $\varepsilon_{t+1}$ é serialmente não-correlacionado. Já que toda nova informação referente a mudanças nos preços à vista futuros é imediatamente refletida em mudanças nos preços futuros correntes, o coeficiente b deve ser não zero. E como a informação passada já foi incorporada nos preços futuros correntes, os coeficientes das mudanças nos preços defasados à vista e futuro, $\beta_{i} \mathrm{e} \gamma_{i}$ devem ser zero. Se as restrições não se verificam os mercados futuros são ineficientes, visto que é possível predizer $S_{t+1}$ com base nos preços à vista passados e nos preços futuros correntes (BECK, 1994).

\section{Resultados e Discussão}

Dados do mercado brasileiro do boi gordo foram obtidos da Bolsa de Mercadorias e Futuros (BM\&FBovespa) de São Paulo. As séries diárias de preços futuros e à vista dos contratos cobrem o período de setembro de 2000 a junho de 2004, com um total de 915 observações. As séries de preço estão em nível, pois uma transformação em logaritmos (usual na literatura de mercados futuros) pode invalidar os resultados quando $c_{0} \neq 0$ e $c_{1} \neq 1$, como estamos assumindo. 
Inicialmente, foi examinada cada uma das séries de preços para verificar se as mesmas são processos estacionários. O teste de Dickey-Fuller Aumentado (DICKEY e FULLER, 1981), ou teste ADF, foi usado para determinar o número de raízes unitárias das séries de preço. Se as duas séries forem não-estacionárias, pode-se testar a co-integração e, caso sejam co-integradas, pode-se testar restrições sobre os parâmetros da Equação (1). Os critérios considerados na determinação do número de defasagens foram os critérios de informação de Akaike (AIC) e de Schwarz (SBC), o teste do multiplicador de Lagrange (teste LM) e o teste de Ljung-Box (teste LB). O número ótimo de defasagens resultante em cada critério foi: $\mathrm{AIC}=7 ; \mathrm{LB}=7 ; \mathrm{LM}=5$; e $\mathrm{SBC}=3$. $\mathrm{O}$ teste $\mathrm{ADF}$ foi realizado com 7 defasagens, já que o teste LB é considerado mais robusto. A aplicação do teste ADF, como implementado no programa RATS, não permite rejeitar a hipótese nula de existência de raiz unitária. Os resultados são apresentados na Tabela 1. O teste ADF da primeira diferença de cada uma das variáveis (não reportado aqui) rejeitou a hipótese nula de raiz unitária. Isso sugere que as séries de preços futuros e à vista do boi gordo podem ser consideradas processos não-estacionários de ordem um, I(1).

Tabela 1. Teste de raiz unitária.

\begin{tabular}{cc}
\hline Preços & $\begin{array}{c}\text { Estatística } \mathbf{t} \\
\text { para o teste ADF }\end{array}$ \\
\hline À vista & -0.4681 \\
Futuros & -0.5543 \\
\hline
\end{tabular}

Fonte: Resultados da pesquisa.

Valor crítico ao NS de $5 \%=-2,86$.

Dado que as variáveis são integradas de mesma ordem, o próximo passo é realizar o teste de co-integração e verificar se os resíduos são estacionários. As séries são co-integradas se $\mu_{t+1}=S_{t+1}-c_{0}-c_{1} F_{t}$ é uma série estacionária, o que implica que $S_{t+1}$ e $F_{t}$ não se movem muito longe uma da outra embora ambas sejam não-estacionárias. $O$ teste $\mathrm{ADF}$ usado para verificar a estacionariedade dos resíduos é conhecido como teste de Engle-Granger aumentado. Se os resíduos forem integrados de ordem zero, I(0), são estacionários (não tem raiz unitária) e as séries são co-integradas. Para testar a estacionaridade dos resíduos considerando co-integração, devem ser usados os valores críticos estabelecidos por MacKinnon (1996), visto que os valores estabelecidos para os testes ADF não são mais válidos. Os resultados obtidos indicam que as duas séries têm uma raiz unitária e os resíduos são I(0), pois a estatística tau calculada foi de $-8,583$, para um valor crítico de $-3,410$. Logo, as séries de preço futuro e à vista, apesar de individualmente serem não-estacionárias, são co-integradas. 
Os resultados relevantes da regressão de co-integração, realizada com o preço à vista como variável dependente, foram:

\begin{tabular}{cccc}
$\begin{array}{c}\text { Variável } \\
\text { Dependente }\end{array}$ & $\begin{array}{c}\text { Regressão de } \\
\text { Co-integração }\end{array}$ & R $^{2}$ Ajustado & $\begin{array}{c}\text { Estatística } t \\
\text { para o teste ADF }\end{array}$ \\
\hline$S_{t+1}$ & $S_{t+1}=1,262+0,974 F_{t}$ & 0,990 & 305,447
\end{tabular}

A co-integração é somente uma condição necessária para eficiência de mercado. A eficiência de mercado também requer que os preços futuros sejam um estimador não viesado dos preços correntes, i.é., $c_{0}=0$ e $c_{1}=1$ na Equação (1). Restrições no vetor de co-integração foram testadas para os preços co-integrados do boi gordo, usando o procedimento "restrict" do RATS. A restrição conjunta $c_{0}=0$ e $c_{1}=1$, imposta ao vetor de co-integração não foi rejeitada:

$\mathrm{F}(1,912)=66,04793$ com nível de significância 0,00000000.

Conseqüentemente, há evidência para suportar as hipóteses de eficiência de mercado e não viés no longo prazo (o preço futuro é um estimador não viesado do preço à vista), para o mercado do boi gordo da BM\&FBovespa.

O número de defasagens na Equação (5) foi determinado iniciando com um modelo geral com doze defasagens de $\Delta S_{t}$ e $\Delta F_{t}$, e eliminando, em sucessivas estimações, as defasagens não significantes (a 5\%). Isso garante que os resíduos sejam não autocorrelacionados. O termo de erro defasado, $\mu_{t}$, foi recuperado da Equação (1), a regressão de co-integração. O modelo de correção de erros estimado foi:

$$
\begin{aligned}
& \Delta S_{t+1}=0,005-0,02 \mu_{t}+0,066 \Delta F_{t-1}+0,322 \Delta S_{t-1}+0,185 \Delta S_{t-2}+0,042 \Delta F_{t-2}+0,025 \Delta F_{t-3}+0,052 \Delta F_{t-4}
\end{aligned}
$$

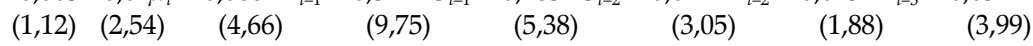

Os números entre parênteses são as estatísticas t. O coeficiente de determinação ajustado $\left(R^{2}{ }_{\text {adj }}\right)$ foi de 0,327 e a estatística Durbin-Watson, de 2,00.

O coeficiente de $\mu_{t}$ foi significantemente diferente de zero a $10 \%$, consistente com os resultados anteriores de que os preços à vista e futuros são co-integrados. Assim, as conclusões sobre a eficiência de mercado e o não viés permanecem válidas. 


\section{Conclusões}

O objetivo deste artigo foi testar a eficiência do mercado futuro brasileiro do boi gordo na presença de um prêmio ao risco. Os resultados empíricos indicaram a presença de raiz unitária nos preços futuros e à vista do boi gordo, o que implica na não-estacionaridade das séries de preços, e assim foi usada a abordagem de co-integração para testar a eficiência de mercado. O teste ADF indicou que os resíduos são estacionários, resultando na não rejeição das hipóteses de que o mercado é eficiente. Para testar a hipótese de que os preços futuros são estimadores não viesados dos preços à vista no longo prazo, foi imposta uma restrição conjunta, $c_{0}=0$ e $c_{1}=1$, ao vetor de co-integração, a qual não foi rejeitada, confirmando a hipótese do não viés. Entretanto, na presença de um prêmio ao risco, a hipótese de não viés pode ser rejeitada mesmo quando o mercado é eficiente. Para testar a eficiência de mercado sem depender da hipótese de que não existe um prêmio ao risco foi usado um modelo de correção de erros. A presença de um prêmio ao risco não causou a rejeição da hipótese de não viés. Conclui-se, portanto, que o mercado futuro do boi gordo da BM\&FBovespa é um mercado eficiente e que no longo prazo os preços futuros são estimadores não viesados dos preços à vista.

Como a literatura tem mostrado a possibilidade de resultados divergentes em função de fatores como, entre outros, período de tempo analisado, sazonalidade, sobreposição de dados e desigualdade de espaçamento entre observações, é possível que conclusões mais robustas sejam obtidas quando alguns de tais fatores são levados em conta. Da mesma forma, diferentes técnicas econométricas (como processos $\mathrm{ARCH}$, por exemplo) podem ser usadas para reforçar ou não os resultados que o presente artigo chegou. Dado que o mercado brasileiro do boi gordo provavelmente já deve ter passado de sua fase imatura, é importante a realização de estudos considerando os fatores acima, para aumentar a compreensão do funcionamento deste mercado. Além disso, o mercado futuro do boi gordo brasileiro tem mostrado um grande crescimento, se mantendo a frente de outros produtos agropecuários nos últimos anos, e tem potencial para crescer ainda mais. Assim, as ferramentas financeiras do mercado futuro provavelmente continuarão a ser cada vez mais utilizadas pelos produtores, já que funcionam como um instrumento de proteção patrimonial e de suporte na busca de maior estabilidade na renda da atividade. A garantia de que este mercado é eficiente - conforme os resultados deste estudo indicam permite maior certeza no estabelecimento do preço do produto pelos pecuaristas, o que também aumenta a eficiência e a rentabilidade do seu negócio. 


\section{Referências Bibliográficas}

ACKER, L. F.; RACINE, M. D. 1999. Stochastic trends and cointegration in the market for equities. Journal of Economics and Business, 51(2): 133-143.

BECK, S. E. 1994. Cointegration and market efficiency in commodity futures markets. Applied Economics, 26, 249-257.

BESSLER, D. A.; COVEY, T. 1991. Cointegration: Some results on U.S. cattle prices. Journal of Futures Markets, 11:461-474.

BM\&F. 2006. Relatório Agropecuário Mensal - Agosto 2006. Bolsa de Mercadorias \& Futuros, São Paulo.

BRESSAN, A. A. 2004. Tomada de decisão em futuros agropecuários com modelos de previsão de séries temporais. RAE-eletrônica, 3(1). Art. 9.

CHU, Q. C.; HSIEH, W. G.; TSE, Y. 1999. Price discovery on the S\&P 500 index markets: An analysis of spot index, index futures, and SPDRs. International Review of Financial Analysis, 8(1): 21-34.

CROWDER, W. J.; PHENGPIS, C. 2003. Testing futures market efficiency using adaptive estimation. University of Texas, Economics Department Working Paper, 7.

DANTHINE, J. P. 1978. Information, futures prices and stabilizing speculation. Journal of Economic Theory, 17: 79-98.

DICKEY, D. A.; FULLER, W. A. 1981. Likelihood ratio statistics for autoregressive time series with a unit root, Econometrica, 49: 1057-1072.

ENGLE, R.; GRANGER, C. 1987. Cointegration and error correction: representation, estimation and testing. Econometrica, 55: 251-276.

FAMA, E.F. 1970. Efficient capital markets: A review of theory and empirical work." Journal of Finance. 25,383-417.

FAMA, E.F.; FRENCH, K. R. 1987. Commodity futures prices: Some evidence on forecast power, premiums and the theory of storage. Journal of Business,60: 55-73.

GARCIA, P.; LEUTHOLD, R. M.; EGELKRAUT, T. M. 2004. Issues and Research Opportunities in Agricultural Futures Markets. In: Symposium on Risk Management in Less Developed and More Developed Countries, Melbourne, 18-19 November 2004.

GRANGER, C. W. J. 1986. Developments in the study of cointegrated economic variables. Oxford Bulletin of Economics and Statistics, 48: 213-228. 
GRANGER, C. W. J. E NEWBOLD, P. 1974. Spurious regression in econometrics. Journal of Econometrics, 2: 111-120.

GRAY, R.W.; RUTLEDGE, D. J. S. 1971. The economics of commodity futures markets: A survey. Review of Marketing and Agricultural Economics. 39: 3-54.

JOHANSEN, S. 1988. Statistical analysis of cointegration vector. Journal of Econometric Dynamic and Control, 12, 231-254.

KELLARD, N.; NEWBOLD, P.; RAYNER, A.; ENNEW, C. 1999. The relative efficiency of commodity futures markets. Journal of Futures Market, 19, 413-432.

LAZZARINI, S. G.; ZYLBERSZTAIN, D.; TAKAKI, F. S. 1998. Inovações contratuais em mercados futuros: o caso do boi gordo na BM\&F. RAC, 2(3): 07-26.

LEUTHOLD, R. M. 1974. The price performance of the futures market of a nonstorable commodity: live beef cattle. American Journal of Agricultural Economics, 56 (2):64-78.

LEUTHOLD. R. M. 1979. An analysis of the futures - cash prices basis for live beef cattle. North Central Journal of Agricultural Economics, 1: 47-52.

MCKENZIE, A. M.; HOLT, M. T. 2002. Market efficiency in agricultural futures markets. Applied Economics. 34,1519-1532.

MACKINNON, J. G. 1996. Numerical distribution functions for unit root and cointegration tests. Journal of Applied Econometrics, 11: 601-618.

MALKIEL, B.G. 2003. The efficient market hypothesis and its critics. Journal of Economic Perspectives. 17: 59-82.

MARTIN, N. B.; BEZERRA, L. M. C. 2005. Mercado futuro do boi gordo: o maior crescimento entre os derivativos agropecuários. Boletim do CCTC-IEA número HP-40/2005.

NEWBOLD, P.; RAYNER, A.; ENNEW, C.; MARROCU, E. 1999a. Testing seasonality and efficiency in commodity futures markets. University of Nottingham, School of Economics Discussion Paper, 33.

NEWBOLD, P.; RAYNER, A.; ENNEW, C.; MARROCU, E. 1999b. Futures markets efficiency: evidence from unevenly spaced contracts. University of Nottingham, School of Economics Discussion Paper, 34.

ZULAUF, C. R.; IRWIN, S. H. 1997. Market efficiency and marketing to enhance income of crop producers. OFOR Economic Paper Series, 97-04. 